\title{
Forecasting Parameters of Earth Surface Subsidence to Assess Safe Operation of Engineering Structures
}

\author{
Vitaliy A.Trofimov ${ }^{1}$, Tamara G. Makeeva ${ }^{2, *}$ \\ ${ }^{1}$ Research Institute of Comprehensive Exploitation of Mineral Resources, Russian Academy of Sci- \\ ences, Moscow, Russia \\ ${ }^{2}$ Moscow state university of civil engineering, Yaroslavskoye shosse, 26, Moscow, Russia, 129337
}

\begin{abstract}
Mining activities, particularly at shallow depths, create heavy environmental impact that causes irreversible changes in the environment's properties, structure and so on. Such unnatural interference frequently makes the environment unusable to an extent in future. It is known that exhaustion of stratified deposits causes notable subsidence of earth surface that may destroy structures, and interrupt the mode of water flows both on surface and underground. To describe the parameters of earth surface subsidence trough under the impact of underground mining of stratified deposits, this paper uses a three-parameter function expressed with the Gauss probability integral. We also propose a numerical algorithm to find the function's parameters based on the values of vertical shift of reference points, measured in situ with benchmark stations. The algorithm is based on the least-squares method, while the minimum value of the respective functionality discrepancies are found with random search. Analysis of the specifics of the function proper helped to propose an approximated method to find its parameters. The paper compares approximating curves produced by both methods, with field measurements.
\end{abstract}

\section{Preamble}

Forecast of earth surface deformations caused by underground mining is today a highly relevant problem yet to be resolved. Correctly evaluated nature of future surface shifts depending on the planned development of underground mining can greatly reduce the damage to above-ground infrastructure, including buildings, roads, pipelines, and other assets that happen to be shifting trough.

Since the middle of the $19^{\text {th }}$ century when the issue was added to the order of business, researchers have proposed and designed a plethora of methods and ways to address the task, based both on the purely empirical approach and on the academic approach related to various mechanical and mathematical models of the rock mass [1]. Each such method is usable to an extent within the existing constraints, which are not however clearly defined. Obviously, the most preferable approach would be one that is simple enough to use, oper-

\footnotetext{
*Corresponding author: makeeva13new@yandex.ru
} 
ates parameters with simple physical meanings that are easily definable and directly related to a specific mining situation; and has a broad enough scope of application.

Let us now assume that the mining situation at the current time is described by a certain existing system of excavations typical for working a stratified deposit and generally with a rather complex configuration both in plane and in depth as multiple strata are mined simultaneously at different depths. Here, earth surface subsidence is controlled by one or more profile lines of reference points on the surface, possibly created at different times and in different areas of the mine field. The objective is to build an algorithm by using data about shifts of reference points (including vertical shifts) and about the state of mining, for some point in time; the algorithm should allow computing earth surface shift parameters, both at a given time and for secondary activities developing in future.

Below, we are going to examine a simplest case of earth surface trough subsidence caused by underground mining. We assume that the following constraints are available and true:

$>\quad$ the mined deposit is a horizontal tabular deposit;

$>\quad$ the overlying rock mass features transversal isotropy with respect to mechanical properties, in particular, resistance to deformation and strength; as a rule, this is related to the stratified nature of the rock mass;

the rock mass, in the rock shifting zone, has no fracturing anomalies, along which mutual shifts of fronts may emerge;

subsidence is caused by only one headway, rectangular in plane, with one side is much larger than the other; in other words, we assume that conditions a flat deformation exists

The situation is illustrated in Figure 1, which shows a cut that runs perpendicular to the long side of the mined space and passes through its middle.

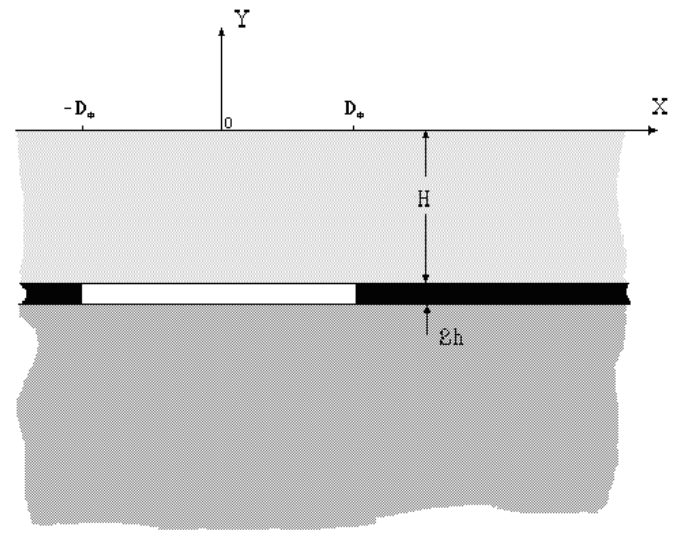

Fig. 1. Schematic representation of a model situation for stratified deposit mining

We need to examine deformation processes in this model setup is necessary to establish that the quantitative description approach represented below for earth surface subsidence is fundamentally valid and applicable. Using the results, in future we can apply the studied principles to more complex situations caused by rejection of certain problem-setting constraints.

Therefore, let us assume that we know the following values found experimentally during field measurements:

location of observatory reference points, described by distance $x_{i}$ along the preset distance from a certain zero point that is frequently but necessarily congruent with the trough center $(i=1,2, \ldots, N, N$-number of reference points on the profile line); 
values of earth surface subsidence $\eta_{i}$ at locations of reference points;

mining parameters, namely:

1. Depth of secondary activities $H$;

2. Half-length of headway $D_{\Phi}$.

\section{Universal Function of Subsidence}

Within the limits of the accepted constraints, it is considered that vertical subsidence of earth surface can be described with function [2]

$$
\eta(x)=-\frac{\eta_{o}}{2}\left[\Phi\left(\frac{D_{p}+x}{C_{p} H}\right)+\Phi\left(\frac{D_{p}-x}{C_{p} H}\right)\right],
$$

which belongs under a three-parameter family, where $\eta_{0}, D_{p}, C_{p}$ - constants typical for



Here: $\eta_{0}$ - value of greatest subsidence of headway roof for complete undermining, typically related to formation thickness $m ; D_{p}$ - parameter that correlates with value $D_{\Phi}$, equal to $\sim 0.7 \div 0.9$ thereof; $C_{p}$ - parameter related to mechanical properties of overlying rock mass.

The main objective is to demonstrate that a type (1) function can be used to approximate with sufficient accuracy experimentally measured values of reference point subsidence. Apparently, the listed numerical parameters are generally unknown in advance in any specific mining situation, and the objective can be resolved by constructing the algorithm proper to define their values.

Real shift values of $\eta_{i}$, differ from the above values $\eta\left(x_{i}\right)$ found under (1) at points $x_{i}$, due to both instrumental errors and local irregularities of the rock mass, and because function (1) corresponds to real-life processes of rock mass deformation. This gives rise to the problem of interpreting all $N$ values of $\eta_{i}$ within model (1).

Therefore, the objective now becomes largely specific and boils down to selecting a type (1) function - in other words, values of $D_{p}, C_{p}, \eta_{o}$ - in a way that all $\eta_{i}$ values lie close to the respective curve (1).

As a measure of proximity, we can use a regulated value brought into the space of point functions, selected partly due to its relative simplicity in the light of analytical research. These requirements are fully met by a quadratic norm

$$
F=\frac{1}{N} \sum_{i}^{N}\left[\eta_{i}-\eta\left(x_{i}\right)\right]^{2}
$$

This is related to the so-called least-squares method. Parameters $D_{p}, C_{p}$, and $\eta_{o}$ should be selected with the least possible functionality $F$. Ultimately, the objective boils down to finding the minimum of function (3) by variables $D_{p}, C_{p}$, and $\eta_{o}$.

A highly efficient and fairly unsophisticated approach to finding the functionality minimum is based on the philosophy of random search methods. This encompasses a multitude of essentially multistep methods, their main feature being that the testing point for the function is selected in a more or less random manner. The core of such methods is that a point is random-selected within a specific area in the space of potential changes of control parameters. The functionality value is computed at this point, and if it is less than a certain input value, the point and its related functionality value are stored in memory as the mini- 
mum and as the input for the next probe. Otherwise, the point is rejected and the next one is selected. Despite its apparent uncertainty, the method is highly efficient for computer-based analysis, and it presents the least requirements to regularity of the function to be examined. By consistently going through the points, we will ultimately arrive at into any small area of the desired solution.

To reduce the required computations, the area of required calculations in the area of possible test values of $D_{p}, C_{p}, \eta_{o}$, from where they are selected with a randomizer, is limited by certain parameters $\Delta_{D}, \Delta_{C}, \Delta_{\eta}$, which set a moving rectangular window of size $2 \Delta_{D} * 2 \Delta_{C} * 2 \Delta_{\eta}$ with its center at the selected test point. The parameter values can be randomized, and their optimal values are the result of a numerical experiment based on the condition of highest convergence rate of the multistep process.

The specifics of the selection window are that it always covers the neighborhood of the current "minimum" point. Random sample here comes only from this fairly small neighborhood, and this significantly narrows down the area of possible search of points, which is practically unlimited from the start. Besides, if the current window fails to yield a point of a smaller value after a certain number of steps (say 1,000), then the window is downsized and the search proceeds in a narrower set.

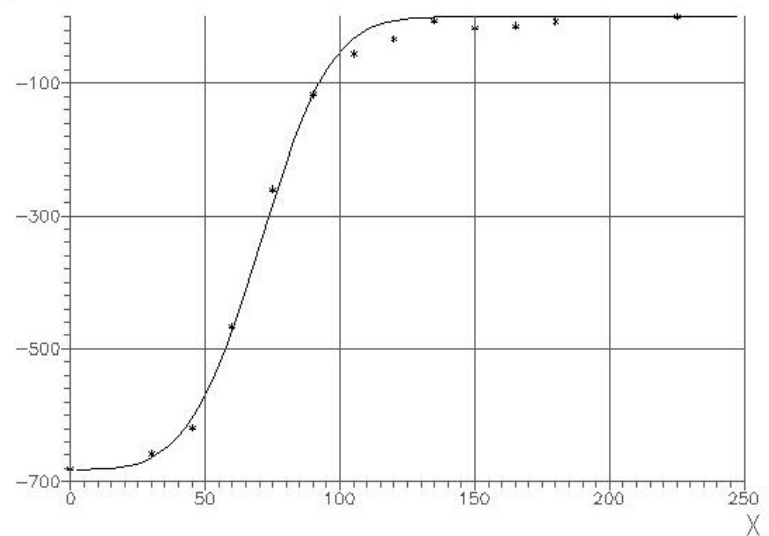

Fig. 2 - Example of approximated field measurements of earth surface subsidence for type (1) functions in incompletely undermined conditions

As a typical example, Figure 2 presents a simulation curve of type (1) and respective experimental data, represented with points, for a real-life case borrowed from the practices of the Donetsk Basin mines, with mining parameters: $H=145 \mathrm{~m}$ and $D_{\Phi}=95 \mathrm{~m}$. Calculations produce the following values of target parameters for function (1): $\eta_{o}=682 \mathrm{~mm}, D_{P}=70 \mathrm{~m}$, $C_{P}=0.144$. Proximity of the corresponding calculated curve to the experimental data is described by magnitude $\sqrt{F}$, which equals $\sim 12 \mathrm{~mm}$.

\section{Approximat Method to Find Subsidence Function Parame- ters}

Proposed below is a different approach to finding the parameters of function (1) based on experimental data, which involves research of certain shared properties of the family of function (1). 
Let us introduce the dimensionless coordinate $\bar{x}$ and dimensionless parameter $\alpha$ into our case

$$
\bar{x}=\frac{x}{D}, \quad \alpha=\frac{C H}{D}
$$

In addition, let us substitute $\eta_{o}$ with the greatest subsidence $V_{\max }$, which for the tabular deposit of the type in question apparently exists at the center of the trough, i.e. for $\bar{x}=0$. It is clearly demonstrated that

$$
\eta_{o}=\frac{V_{\max }}{\Phi\left(\frac{1}{\alpha}\right)}
$$

In our notation, relation (1) should be represented as follows:

$$
v=\frac{V_{\max }}{2 \Phi\left(\frac{1}{\alpha}\right)}\left[\Phi\left(\frac{1-\bar{x}}{\alpha}\right)+\Phi\left(\frac{1+\bar{x}}{\alpha}\right)\right]
$$

By normalizing (6) by $V_{\max }$ and by introducing $\bar{v}=v / V_{\max }$, we ultimately arrive at the relationship for dimensionless value of subsidence in the trough

$$
\bar{v}=\frac{1}{\beta} F(\bar{x}, \alpha)
$$

where

$$
\begin{gathered}
\beta=2 \Phi\left(\frac{1}{\alpha}\right) \\
F(\bar{x}, \alpha)=\Phi\left(\frac{1-\bar{x}}{\alpha}\right)+\Phi\left(\frac{1+\bar{x}}{\alpha}\right)
\end{gathered}
$$

Therefore the initial relationship (1) is represented as dimensionless, thus it is demonstrated that all vertical subsidence movements of earth surface, since they are described with equation (1), regardless the values of real-life mining parameters, they form a single-parameter family derivative from parameter $\alpha$. The appearance of the family (7) is illustrated in Fig. 3, where the values of parameter $\alpha$ are shown with numbers on the respective curve lines.

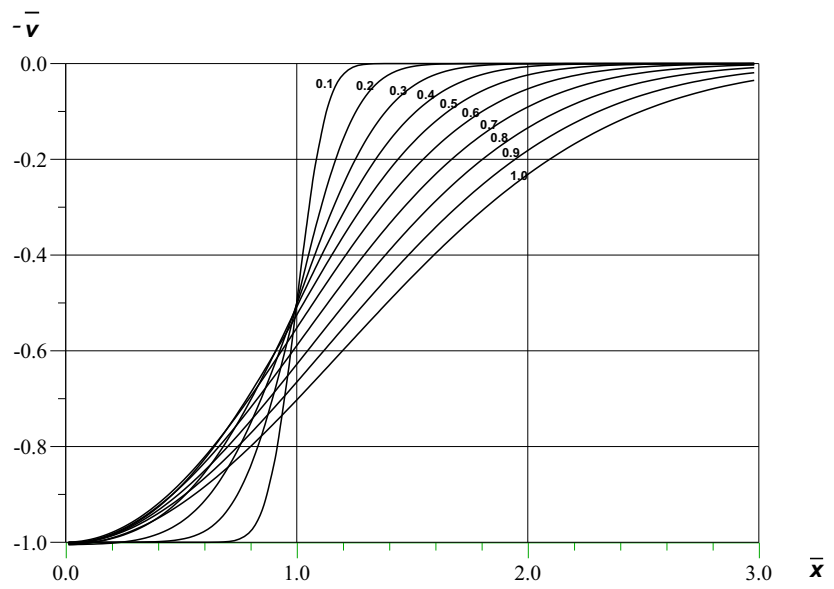

Fig. 3. Dimensionless curves of earth surface subsidence for various values of $\alpha$

Now we examine the dependence of $\bar{v}$ on $\alpha$ for $\bar{x}=1$. Let us note that $\bar{x}=1$ corresponds to parameter value $x=D$. Considering (8), (9), equation (7) gives 


$$
\bar{v}_{l}=\left.\bar{v}\right|_{\bar{x}=I}=\frac{\Phi\left(\frac{2}{\alpha}\right)}{2 \Phi\left(\frac{1}{\alpha}\right)}
$$

The graph represented in Fig. 4 shows that within the change limits of $0 \leq \alpha \leq 0.5$, the value of $\bar{v}_{1}$ is found to be a constant equal to 0.5 , with accuracy of up to $5 \%$. This is because for $\alpha<0.5$ we have $\Phi\left(\frac{1}{\alpha}\right) \cong \Phi\left(\frac{2}{\alpha}\right) \cong 1$. This in turn means that within preset limits all graphs with $\alpha$ virtually intersect at the same point, if $\bar{x}=1$, while $\bar{v}=0.5$. In other words, if $x=D$, earth surface subsidence will be half of the maximum reached at the center of the trough.

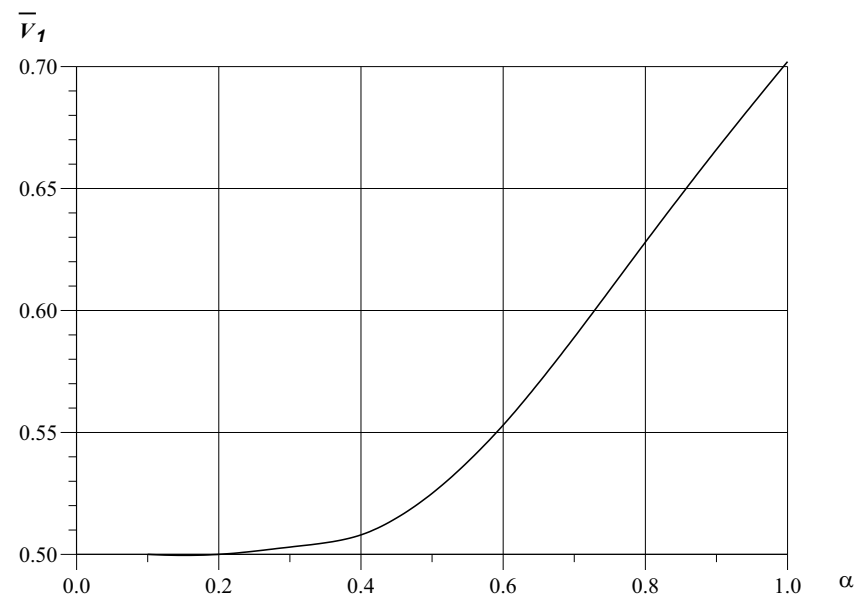

Fig. 4. Dimensionless subsidence of earth surface for $\bar{x}=1$.

Let us now examine the inclination of subsidence curves of (7) for $\bar{x}=1$. We differentiate the relation (7) by $\bar{x}$ and introduce this

finally arriving at

$$
\operatorname{tg} \theta=\left.\frac{\hat{\alpha}}{\hat{\alpha x}}\right|_{\bar{x}=1}
$$

$$
\operatorname{tg} \theta=\frac{1}{\sqrt{2 \pi}} \frac{1}{\alpha \Phi\left(\frac{1}{\alpha}\right)}\left(e^{-\frac{2}{\alpha^{2}}}-1\right)
$$

Therefore, our analysis has established biunique functionality dependencies $\bar{v}_{l}(\alpha)$ and $\operatorname{tg} \theta(\alpha)$, i.e. dimensionless value of earth surface subsidence and inclination for $x=D$ are unambiguously determined by parameter $\alpha$ and reversely (also considering the constraints of $\alpha$ ).

Now we use our dependencies to find the parameters of $\eta_{o}, D$, and $C$ in (1) based on data produced during field measurements of earth surface subsidence at the shifting trough. To this end, we can use the following sequence of steps:

Figures 7 and 8 illustrate how the proposed methodology is used to process test data harvested from a Donbass mine. Measured values of earth surface subsidence are points on the graphs, while $V_{\max }=380 \mathrm{~mm}$. The formation depth is $H=200 \mathrm{~m}$. As is obvious from Fig. 3 and as explained above, $D=84.3 \mathrm{~m}$. Fig. 7 represents actual data of the benchmark station line; Fig. 8 shows normalized curves. 

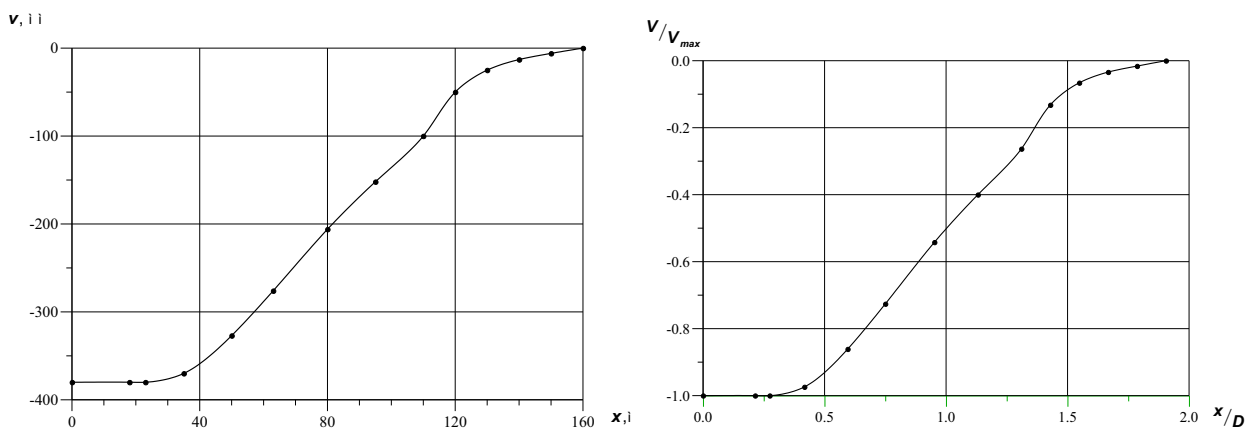

Fig. 5. Actual data on earth surface subsidence from benchmark station line

Fig. 6. Normalized subsidence curve

Directly measured on the graph (Fig.6), to the scale of the axis, we can find that $\operatorname{tg} \theta=0.885$. From this, we find parameter $\alpha=0.465$ and then correspondingly $C=0.196$. After this, we can easily find the greatest value of subsidence for complete undermining case as $\eta_{0}=393 \mathrm{~mm}$.

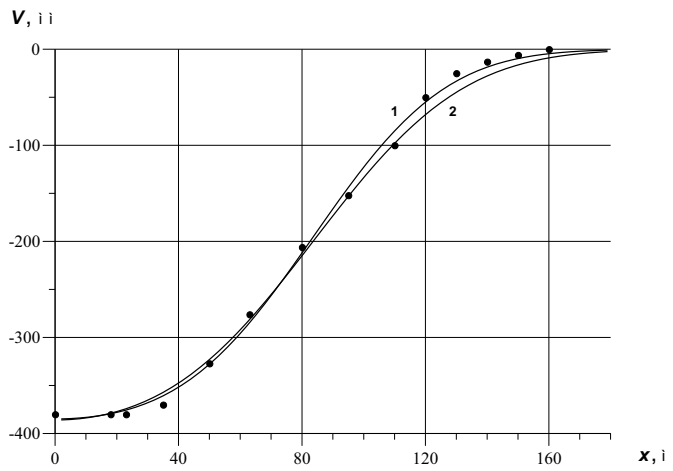

Fig. 7. Comparing earth surface subsidence curves found with different methods, and experimental data

Figure 7 contains comparable experimental data (marked as points), the subsidence curve of type (1) with parameters found through the methodology described above (curve No. 2) and the subsidence curve of type (1), that best approximates the experimental data (curve No. 1, for which $D=82.3 \mathrm{~m}, C=0.18$, and $\eta_{0}=395 \mathrm{~mm}$ ), build using the random search method described above.

Therefore, it has been demonstrated that relation (1) can be used to describe subsidence troughs in the event of flat deformation scenario, achieved by stated algorithms used to find the parameters of function (1).

By differentiating relation (1) we can find angles of inclination of the surface and its longitudinal forces of stretching-compression; this is highly important for safe operation of structures built above ground.

\section{References}

1. G.Cratch, Sdvijenie gornih porod i zaschita podrabatyvaemih soorujeniy (Movement of rocks and protection of underworked structures). Moscow: Nedra, (1978)

2. R.A. Muller, Vliyanie gornih virabotok na deformatsiu zemnoy poverhnosty (Influence of mine workings on deformation of the earth's surface). Moscow: Ugletehizdat, (1958) 